\title{
Least-Squares Interpolation of Fused MR and CT Images in the Wavelet Domain
}

\author{
Eman Sultan, Sayed El- \\ Rabaie, and Fathi E. Abd \\ El-Samie \\ Dept. of Electronics \& Electrical \\ Comm. \\ Faculty of Electronic \\ Engineering, \\ Menofia University, Menouf, \\ 32952, Egypt
}

\author{
Nawal El-Fishawy \\ Dept. of Computer Science \& \\ Engineering, \\ Faculty of Electronic \\ Engineering \\ Menofia University, \\ 32952,Egypt
}

\author{
Said E. El-Khamy \\ Dept. of Electrical Engineering, \\ Faculty of Engineering, \\ Alexandria University, Egypt
}

\begin{abstract}
A suggested approach is presented in this paper to obtain high-resolution images from the fusion and then interpolation of Magnetic Resonance (MR) and Computed Tomography (CT) images. MR and CT images are fused with either the Discrete Wavelet Transform (DWT) or the curvelet transform. After that, a least-squares interpolation step is carried out on the wavelet sub-bands of the fusion result. Simulation results show the feasibility of the fusion process to obtain images with more details and the efficiency of interpolation to obtain high-resolution images.
\end{abstract}

\section{General Terms}

Image processing.

\section{Keywords}

Image interpolation, Image fusion, and Curvelet transform.

\section{INTRODUCTION}

Image fusion is considered as an integration of different source images to produce an image with higher visual quality than the source images. Image fusion is a very important topic for a wide variety of applications [1-5]. It has applications in medical imaging, remote sensing, and digital photographs. It is very important for medical applications, because images of different modalities for the same regions are available, and the fusion of these images will help in obtaining more useful detail information. A further interpolation step on the fused images will be important, if certain regions of them need to be magnified.

Medical imaging has become a vital component of a large number of applications including diagnosis, research, and treatment. In order to provide more accurate clinical information for physicians to deal with medical diagnosis and evaluation, multimodality medical images are needed such as X-ray, CT, MR, Magnetic Resonance Angiography (MRA), and Positron Emission Tomography (PET) images [6]. These multimodality medical images usually provide complementary and occasionally conflicting information. For example, the CT image can provide dense structures like bones and implants with less distortion, but it cannot detect physiological changes, while the MR image can provide normal and pathological soft tissues information, but it cannot support the bone information. In this case, only one kind of image may not be sufficient to provide accurate clinical requirements for the physicians. Therefore, the fusion of the multimodal medical images is necessary, and it has become a promising and very challenging research area in recent years [7-10].

The fusion of MR and CT images of the same region or organ would result in an integrated image of much more details. Researchers have made some attempts for the fusion of MR and CT images. Most of these attempts are directed towards the application of the DWT for this purpose [11-13]. Due to the limited capability of the DWT to deal with images with curved structures, the application of the curvelet transform for this purpose gives better results [14]. The application of the curvelet transform for the fusion of MR and CT images, and an efficient implementation of the inverse interpolation techniques to obtain high-resolution versions from the fused images, were presented in [15].

A digital image interpolation method that is performed in the wavelet domain with a least-squares algorithm was presented in [16]. This method estimates wavelet coefficients in the high-frequency sub-images of the estimated high-resolution image from the low-resolution image using a least-squares algorithm. An inverse wavelet transform is then performed for the synthesis of the HR image. This algorithm is based on interpolating each block, separately in an adaptive manner. It considers both the mathematical model by which the image is acquired and the local activity levels of the block to be interpolated, and performs the interpolation in a small number of iterations.

In this paper, we present a comparison between the curvelet transform and the wavelet transform for the fusion of MR and CT images, and an efficient implementation of the waveletbased image interpolation with the least-squares algorithm to obtain high-resolution images from the fusion results. The paper is organized as follows. Section 2 reviews the wavelet fusion technique with concentration on its limitations and also the curvelet fusion technique. In section 3, the least-squares image interpolation algorithm is reviewed. Section 4 introduces the suggested wavelet-based image interpolation method. Section 5 gives the image quality metrics that can be used for quality assessment of fusion and interpolation results. Section 6 gives the experimental fusion and interpolation results. Finally, section 7 gives the concluding remarks.

\section{THE IMAGE FUSION TECHNIQUES}

Several techniques have been presented for image fusion and an overview of these methods is given in [17]. In this paper, we will concentrate on the wavelet and the curvelet fusion techniques. 


\subsection{Discrete Wavelet Image fusion}

In this sub-section, to better understand the concept and procedure of the wavelet-based fusion technique, a schematic diagram is given in Figure 1. In general, the basic idea of image fusion based on wavelet transform is to perform a multi-resolution decomposition on each source image. The coefficients of both the low-frequency band (approximation band) and high-frequency bands (details bands) of the images to be fused are then merged with a certain fusion rule as displayed in the middle block of Figure 1. The widely-used fusion rule is the maximum selection. This simple rule just selects the largest absolute value for each wavelet coefficient at each location from the input images as the coefficient at the location in the fused image. After that, the fused image is obtained by performing the Inverse DWT (IDWT) of the corresponding combined wavelet coefficients. Therefore, as shown in Figure 1, the detailed fusion steps based on the wavelet transform can be summarized below:

Step 1. The images to be fused must be registered to assure that the corresponding pixels are aligned.

Step 2. These images are decomposed into the wavelet domain.

Step 3. A certain fusion rule is performed to produce the transform coefficients of the fused image.

Step 4. The fused image is constructed by performing an inverse wavelet transform based on the combined transform coefficients from Step 3.

The most frequently used rule is the maximumfrequency rule, which selects the maximum coefficients from the wavelet transformed images [13]. Then, the inverse wavelet transform $\omega^{-1}$ is computed, and the fused image $I(x, y)$ is reconstructed [5]:

$$
I(x, y)=\omega^{-1}\left(\phi\left(\omega\left(I_{1}(x, y)\right), \omega\left(I_{2}(x, y)\right)\right)\right) \text {. }
$$

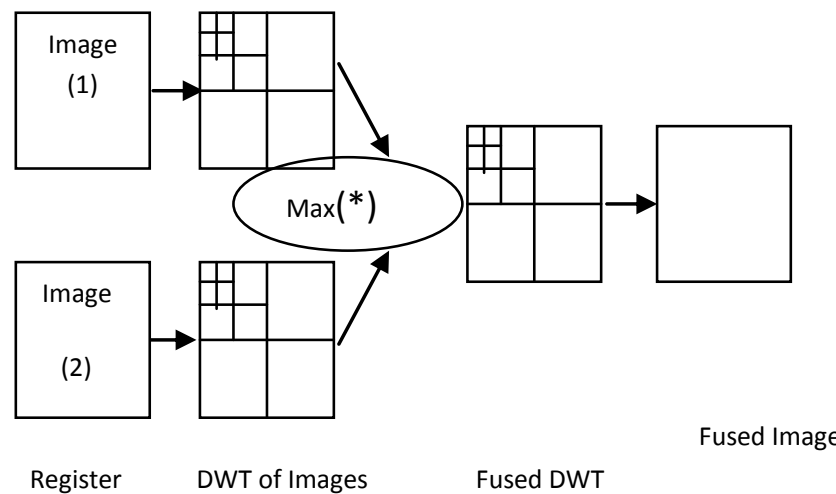

Fig 1 Wavelet fusion.

For long curved edges, the wavelet transform has limited accuracy for edge localization. So, there is an alternative approach, which has a high accuracy of curve localization such as the curvelet transform [14].

\subsection{Curvelet Image Fusion}

The DWT has been one of the most famous tools for image and signal analysis, because of its advantageous property that helps to localize point singularities in a signal or an image. One major disadvantage of the DWT in image processing is that it gives a large number of coefficients in all scales corresponding to the edges of the image. So, in order to exactly reconstruct the edges in an image, several coefficients are required in order to exactly reconstruct the edges in an image. This makes the DWT inefficient for handling long curved edges. Recent approaches such as the ridgelet transform and the curvelet transform are more efficient in handling long linear and curvilinear singularities in an image [18-22].

In the curvelet transform, the Additive Wavelet Transform (AWT) is used instead of the DWT to decompose the image into different sub-bands called the detail planes and the approximation plane, and each sub-band of the detail planes is then partitioned into small tiles. Then, the ridgelet transform is applied on each tile [23]. In this way, the image edges can be represented efficiently by the ridgelet transform, because the image edges will now be almost like small straight lines. Thus, the curvelet transform is considered as an effectively extension of the ridgelet transform to detect curved edges. The algorithm of the curvelet transform can be summarized in the following steps [18-24]:

1. The image is split up into three sub-bands $\Delta_{1}, \Delta_{2}, \Delta_{3}$, and $\mathrm{P}_{3}$ using the AWT, see Figure 2 .

2. The sub-bands $\Delta_{1}, \Delta_{2}$, and $\Delta_{3}$ are divided into small blocks called tiles.

3. The discrete ridgelet transform is performed on each tile of the sub-bands $\Delta_{1}, \Delta_{2}$, and $\Delta_{3}$.

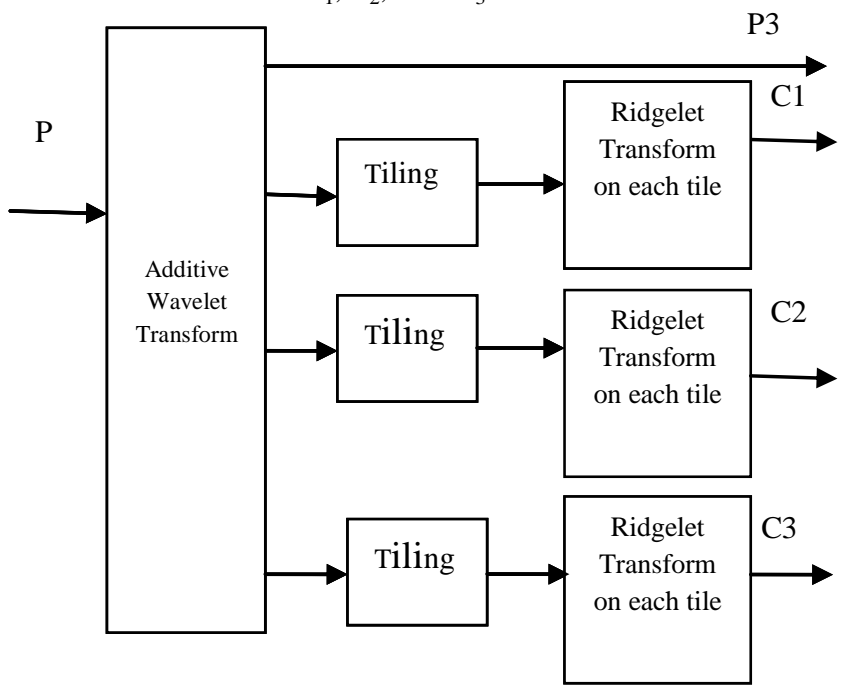

Fig 2 Curvelet transform.

Figure 3 shows a schematic diagram of the curvelet transform steps. A detailed description of these steps is presented in the following sub-sections.

The steps of the curvelet fusion approach of MR and CT images [14] can be summarized as follows:

1. The MR and the CT images are registered.

2. The AWT is performed to spilt both images into three sub-bands.

3. Tiling is performed on the sub-bands $\Delta_{1}, \Delta_{2}$, and $\Delta_{3}$ of each image.

4. The maximum-frequency fusion rule is used for the fusion of the ridgelet transforms of the tiled subbands.

5. An inverse curvelet transform is performed by the AWT reconstruction as shown in Figure 4.

6. A post-processing step can be performed by using a high-pass filter to sharpen the fusion result, if there is some blurring due to the approximation of the digital ridgelet transform.

7. These steps are expected to merge the details in both images into a single image with much more quality. 
The objective of post processing is to enhance edges in the fusion results. This step can be accomplished through the use of a high-pass filter mask $\mathrm{H}_{\mathrm{F}}$ such as [23]:

$$
H_{F}=\left[\begin{array}{ccc}
0 & -1 & 0 \\
-1 & 5 & -1 \\
0 & -1 & 0
\end{array}\right]
$$

$\mathrm{f}\left(\mathrm{n}_{1}, \mathrm{n}_{2}\right)$ $\mathrm{P}_{1}\left(\mathrm{n}_{1}, \mathrm{n}_{2}\right)$ $\mathrm{P}_{2}\left(\mathrm{n}_{1}, \mathrm{n}_{2}\right)$ $\mathrm{P}_{3}\left(\mathrm{n}_{1}, \mathrm{n}_{2}\right)$

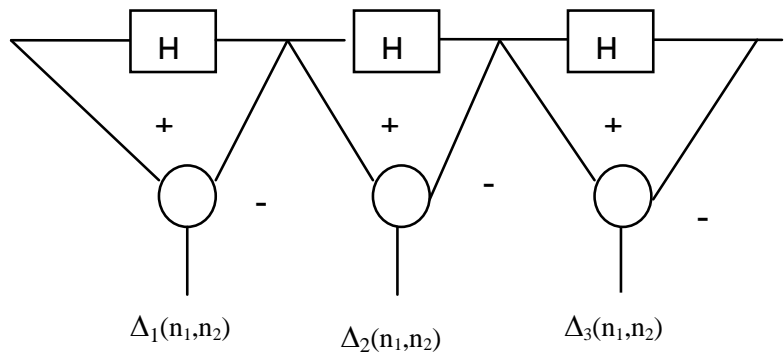

Fig 3 AWT decomposition.

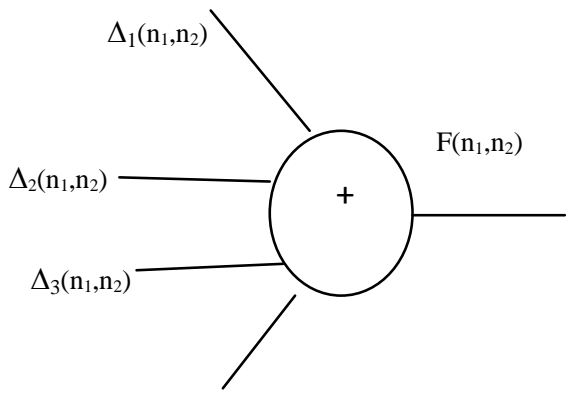

$\mathrm{P}_{3}\left(\mathrm{n}_{1}, \mathrm{n}_{2}\right)$

$\mathrm{F}\left(\mathrm{n}_{1}, \mathrm{n}_{2}\right)=\mathrm{P}_{3}\left(\mathrm{n}_{1}, \mathrm{n}_{2}\right)+\Delta_{1}\left(\mathrm{n}_{1}, \mathrm{n}_{2}\right)+\Delta_{2}\left(\mathrm{n}_{1}, \mathrm{n}_{2}\right)+\Delta_{3}\left(\mathrm{n}_{1}, \mathrm{n}_{2}\right)$

Fig 4 AWT reconstruction.

\section{ADAPTIVE LEAST-SQUARES IMAGE INTERPOLATION}

In the adaptive least-squares interpolation algorithm, the image to be interpolated is divided into small overlapping blocks of size $\mathrm{M} \times \mathrm{M}$, and the objective is to obtain an interpolated version of each block of size $\mathrm{N} \times \mathrm{N}$. The relation between the available LR and the estimated HR block is given by [25]:

$\hat{\mathbf{f}}_{\mathbf{i}, \mathbf{j}}=\mathbf{W g}_{\mathbf{i}, \mathbf{j}}$

where $\mathbf{g}_{\mathbf{i}, \mathbf{j}}$ and $\hat{\mathbf{f}}_{\mathbf{i}, \mathbf{j}}$ are the $\mathbf{M}^{2} \times 1$ and $\mathrm{N}^{2} \times 1$ lexicographically-ordered low-resolution, and the estimated high-resolution blocks at position $(i, j)$, respectively. $\mathbf{W}$ is the $\mathrm{N}^{2} \times \mathrm{M}^{2}$ weight matrix required to obtain the high-resolution block from the low-resolution block. This matrix is required to be adaptive from block to block to accommodate for the local activity levels of each block. By using Eq. 3, we can get the least-squares solution by minimizing the Mean Square Error (MSE) of estimation as follows :
$\boldsymbol{\Psi}=\left\|\mathbf{f}_{\mathbf{i}, \mathbf{j}}-\hat{\mathbf{f}}_{\mathbf{i}, \mathbf{j}}\right\|^{2}=\left\|\mathbf{f}_{\mathbf{i}, \mathbf{j}}-\mathbf{W} \mathbf{g}_{\mathbf{i}, \mathbf{j}}\right\|^{2}$

Differentiating both sides of Eq.(4) with respect to $\mathbf{W}$ gives:

$$
\frac{\partial \Psi}{\partial \mathbf{W}}=-2\left(\mathbf{f}_{i, j}-\hat{\mathbf{f}}_{i, j}\right)\left(\mathbf{g}_{\mathrm{i}, \mathrm{j}}\right)^{t}
$$

This minimization leads directly to the following solution for $\mathbf{W}$ as follows :

$$
\mathbf{W}^{k+1}=\mathbf{W}^{k}-\eta\left[\frac{\partial \Psi}{\partial \mathbf{W}}\right]^{k}=\mathbf{W}^{k}+\mu\left(\mathbf{f}_{i, j}-\hat{\mathbf{f}}_{i, j}^{k}\right)\left(\mathbf{g}_{i, j}^{k}\right)^{t}
$$

where $\eta$ is a constant and $\mu$ is the convergence parameter.

Using the above equation in estimating the weight matrix W requires the samples of the original high-resolution block $\mathbf{f}_{\mathbf{i}, \mathbf{j}}$ to be known, which is not practical. The following equation relates the available low-resolution block to the original high-resolution block.

$$
\mathbf{g}_{i, j}=\mathbf{D f}_{i, j}
$$

The matrix $\mathbf{D}$, which is called the decimation matrix, is of size $\mathrm{M}^{2} \times \mathrm{N}^{2}$. Thus, the following cost function needs to be minimized [12]:

$$
\boldsymbol{\Phi}=\left\|\mathbf{D}\left(\mathbf{f}_{\mathrm{i}, \mathrm{j}}-\hat{\mathbf{f}}_{\mathrm{i}, \mathrm{j}}\right)\right\|^{2}
$$

The above equation means minimizing the MSE between the available low-resolution block and a downsampled version of the estimated high-resolution block. This leads to:

$$
\boldsymbol{\Phi}=\left\|\mathbf{g}_{i, j}-\mathbf{D} \hat{\mathbf{f}}_{\mathrm{i}, \mathrm{j}}\right\|^{2}=\left\|\mathbf{g}_{\mathrm{i}, \mathrm{j}}-\mathbf{D W} \mathbf{g}_{\mathrm{i}, \mathrm{j}}\right\|^{2}
$$

Differentiating Eq. (9) with respect to $\mathbf{W}$ and using Eq.(3) leads to:

$$
\frac{\partial \Phi}{\partial \mathbf{W}}=-2 \mathbf{D}^{t}\left(\mathbf{g}_{i, j}-\mathbf{D}_{i, j}\right)\left(\mathbf{g}_{i, j}\right)^{t}
$$

Using Eq.(10), the weight matrix can be adapted using the following equation:

$$
\mathbf{W}^{k+1}=\mathbf{W}^{k}-\eta\left[\frac{\partial \Phi}{\partial \mathbf{W}}\right]^{k}=\mathbf{W}^{k}+\mu \mathbf{D}^{t}\left(\mathbf{g}_{i, j}-\mathbf{D}_{\mathrm{i}, \mathrm{j}}^{k}\right)\left(\mathbf{g}_{\mathrm{i}, \mathrm{j}}^{k}\right)^{t}
$$

The adaptation of Eq.(11) can be easily performed, since it does not require the original high-resolution block to be known a priori.

\section{WAVELET IMAGE INTERPOLATION}

The wavelet-based image interpolation method [16] depends on the decomposition of the low-resolution image into an approximation component and three detail components. The available low-resolution image is considered as the approximation component of the required high-resolution image. The detail components of the high-resolution image are derived from the detail components of the low-resolution image with a least-squares algorithm. The terms $\mathbf{g}_{i, j}$ and $\hat{\mathbf{f}}_{\mathrm{i}, \mathrm{j}}$ in Eq. (11) represent the blocks of the detail sub-bands of the low-resolution image, and the blocks of the detail subbands of the estimated high-resolution sub-bands. The interpolation algorithm will be implemented in an iterative manner to reduce the MSE between the original and 
interpolated images. Figure (5) shows the proposed interpolation method.

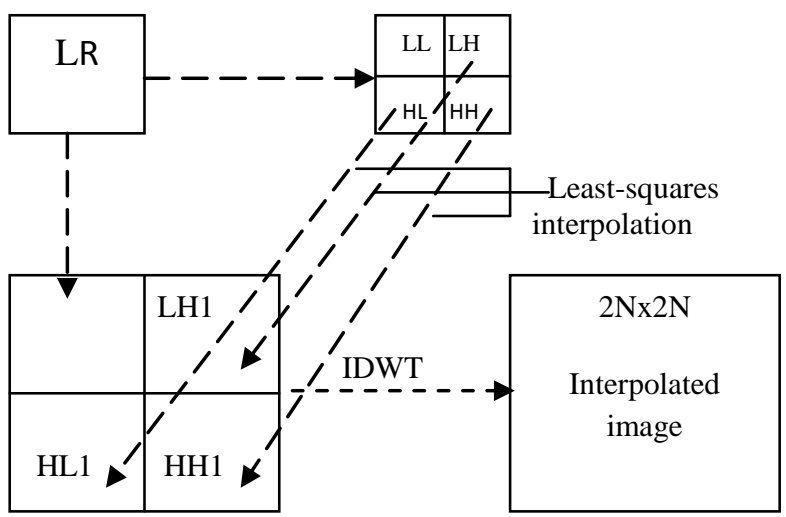

Fig 5 Wavelet- based image interpolation with a leastsquares algorithm.

\section{IMAGE QUALITY METRICES}

In our study, subjective assessment of image quality is performed by measuring the Peak Signal-to-Noise Ratio (PSNR) of the interpolated images: $R M S E=\sqrt{\frac{\sum_{i=1}^{M} \sum_{j=1}^{N}[R(i, j)-F(i, j)]^{2}}{M \times N}}$

where $R(i, j)$ is the original image and $F(i, j)$ is the reconstructed image. The smaller the value of the Root Mean Square Error (RMSE), the better the fusion performance. The formula for PSNR is given by [26]:

$$
P S N R=10 \times \log \left(\left(f_{\max }\right)^{2} / R M S E^{2}\right)
$$

where $f_{\max }$ is the maximum gray-scale value of the pixels in the reconstructed image. The higher the value of the PSNR, the better the performance of the used algorithm.

Another quantitative measured is the similarity of edges (S) [14]. The steps for calculating this similarity are as follows:

1. Edge detection of the reconstructed image using a suitable edge detector such as the Canny detector.

2. Edge detection of the original image

3. Estimation of the ratio between the similar edge pixels between the reconstructed image and the original image and the total number of edge pixels.

\section{RESULTS AND DISCUSSION}

In this section, two different experiments are carried out to apply the wavelet-based image interpolation with the leastsquares algorithm to the original CT image, the original MR image, and the fused MR and CT images by both the wavelet and curvelet fusion techniques.

Unfortunately, in image interpolation, there is no reference image to measure the PSNR values and the similarity values of the obtained results with it. To solve this problem and compare between the interpolation of the original images and the interpolation of the fused image [16], we can decimate the original MR, and CT images prior to fusion and compare with these original images.

In our two experiments we will follow the following procedure:
1. Image 1 (MR image) is down-sampled and then interpolated.

2. Image 2 (CT image) is down-sampled and then interpolated.

3. Down-sampled versions of images 1 and 2 are wavelet fused.

4. Down-sampled versions of images 1 and 2 are curvelet fused. For the curvelet fusion, small tiles of dimensions $12 \times 12$ with two pixels of overlapping from each side are utilized to approximate long curved lines by small straight lines and to avoid the edge effects. A high-pass filtering step is used to sharpen the curvelet fused images.

5. The fused image is interpolated.

Tables 1 and 2 give the evaluation metrics for interpolation results.

Table 1 PSNR values for interpolation results.

\begin{tabular}{|c|c|c|c|}
\hline \multicolumn{2}{|c|}{ Images } & \multirow{2}{*}{$\begin{array}{c}\begin{array}{c}\text { Similarity } \\
\text { Type }\end{array} \\
S_{C} \\
\end{array}$} & \multirow{2}{*}{$\begin{array}{c}\begin{array}{c}\text { Similarity } \\
\text { Value }\end{array} \\
0.0754 \\
\end{array}$} \\
\hline \multirow{4}{*}{$\begin{array}{l}\text { First } \\
\text { case }\end{array}$} & \multirow{2}{*}{ Wavelet } & & \\
\hline & & $S_{M}$ & 0.1109 \\
\hline & \multirow{2}{*}{ Curvelet } & $S_{C}$ & 0.1719 \\
\hline & & $S_{M}$ & 0.2041 \\
\hline \multirow{4}{*}{$\begin{array}{l}\text { Second } \\
\text { case }\end{array}$} & \multirow{2}{*}{ Wavelet } & $S_{C}$ & 0.0951 \\
\hline & & $S_{M}$ & 0.0674 \\
\hline & \multirow{2}{*}{ Curvelet } & $S_{C}$ & 0.2124 \\
\hline & & $S_{M}$ & 0.1376 \\
\hline \multicolumn{3}{|c|}{ Image to be interpolated } & PSNR (dB) \\
\hline \multirow{4}{*}{$\begin{array}{l}\text { First } \\
\text { case }\end{array}$} & \multicolumn{2}{|c|}{ Image 1} & 26.2858 \\
\hline & \multicolumn{2}{|c|}{ Image 2} & 29.6043 \\
\hline & \multicolumn{2}{|c|}{ Wavelet fusion result } & 27.1586 \\
\hline & \multicolumn{2}{|c|}{ Curvelet fusion result } & 30.7028 \\
\hline \multirow{4}{*}{$\begin{array}{l}\text { Second } \\
\text { case }\end{array}$} & \multicolumn{2}{|c|}{ Image 1} & 24.7090 \\
\hline & \multicolumn{2}{|c|}{ Image 2} & 24.7248 \\
\hline & \multicolumn{2}{|c|}{ Wavelet fusion result } & 24.3030 \\
\hline & \multicolumn{2}{|c|}{ Curvelet fusion result } & 26.8167 \\
\hline
\end{tabular}

Table 2 Similarity values for interpolation results.

The obtained results are in favor of curvelet fusion and then interpolation. 


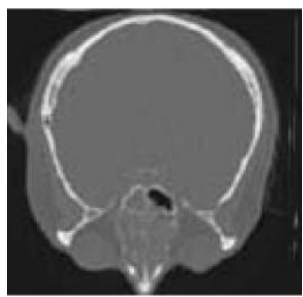

(a) CT image.

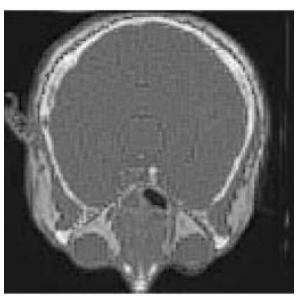

(c) Wavelet fusion.

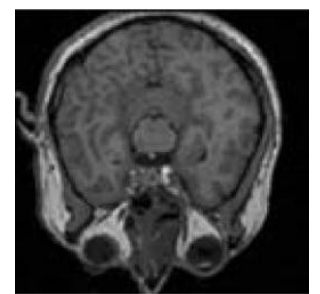

(b) $M \mid R$ image.

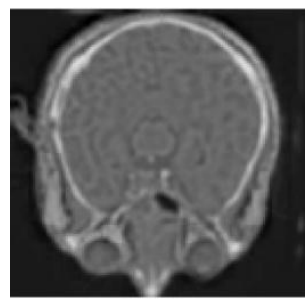

(d) Curvelet fusion.

Fig 6 Fusion of MR and CT images for case 1.

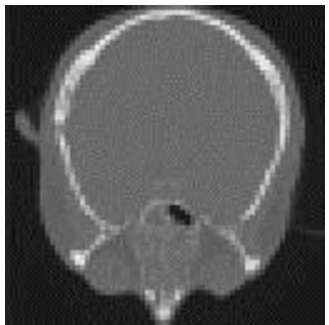

(a) Interpolated CT image.
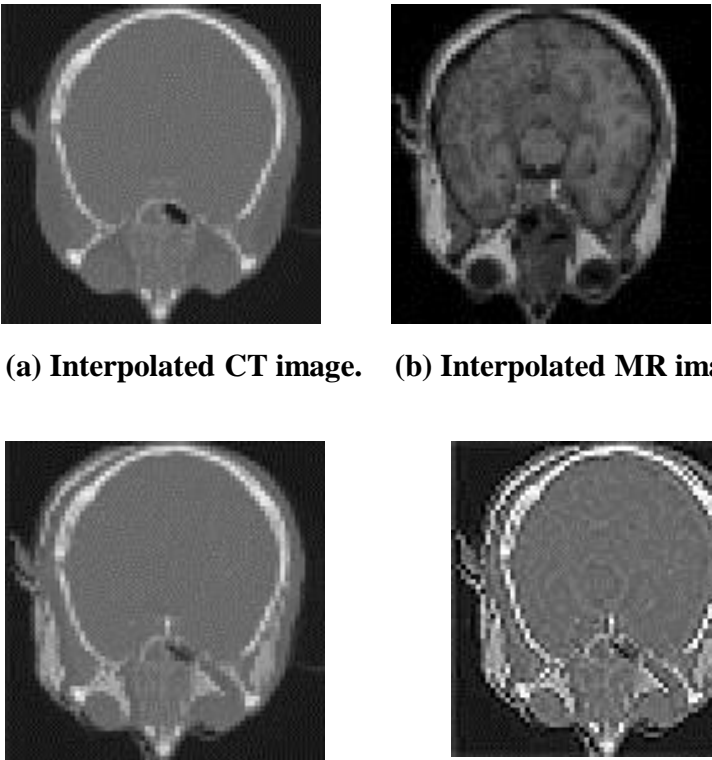

(b) Interpolated MR image.

(c) Interpolated wavelet fusion. (d) Post processing of (c)
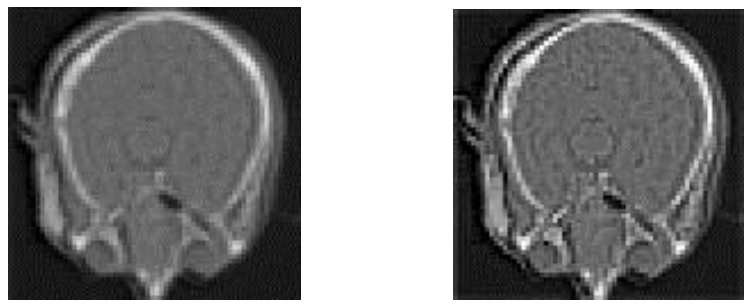

(e) Interpolated curvelet fusion. (f) Post processing of (e).

Fig 7 Interpolation results.

\section{CONCLUSION}

In this paper, we presented an efficient way to obtain highresolution images from the fusion of MR and CT images. The suggested approach adopts a least-squares strategy to build the wavelet sub-bands of the required high-resolution image in an iterative manner. Simulation results show the success of the proposed approach to obtain images with better resolution, especially when the curvelet transform is used to merge the MR and CT images with a final post-processing step.

\section{REFRENCES}

[1] A. Wang, H. J. Sun, and Y. Y. Guan, "The application of wavelet transform to multi-modality medical image fusion," in IEEE International Conference on Networking, Sensing and Control (IEEE, 2006), pp. 270 274.

[2] H. Moustafa and S. Rehan, "Applying image fusion techniques for the detection of hepatic lesions and acute intra-cerebral hemorrhage," in ITI 4th International Conference on Information and Communications Technology 2006 (IEEE, 2006).

[3] C. Pohl and J. L. van Genderen, "Multisensor image fusion in remote sensing: concepts, methods and application,” Int. J. Remote Sens. 19, 823-854 (1998).

[4] A. A. Goshtasby and S. Nikolov, "Image fusion: advances in the state of the art," Inf. Fusion 8, 114-118 (2007).

[5] M. Ouendeno, "Image fusion for improved perception," Ph.D. dissertation (Florida Institute of Technology, 2007).

[6] F. Maes, D. Vandermeulen, and P. Suetens, "Medical image registration using mutual information," Proceedings of the IEEE, vol. 91, no. 10, pp. 1699-1721, 2003.

[7] V. Barra and J.-Y. Boire, "A general framework for the fusion of anatomical and functional medical images," NeuroImage, vol. 13, no. 3, pp. 410-424, 2001.

[8] Y.-M. Zhu and S. M. Cochoff, "An object-oriented framework for medical image registration, fusion, and visualization," Computer Methods and Programs in Biomedicine, vol. 82, no. 3, pp. 258-267, 2006.

[9] V. S. Petrovic and C. S. Xydeas, "Gradient-based multiresolution image fusion," IEEE Transactions on Image Processing, vol. 13, no. 2, pp. 228-237, 2004.

[10] Z. Zhang and R. S. Blum, "A categorization of multiscale-decomposition-based image fusion schemes with a performance study for a digital camera application," Proceedings of the IEEE, vol. 87, no. 8, pp. 1315-1326, 1999.

[11] S. Udomhunsakul and P. Wongsita, "Feature extraction in medical MRI images," in Proceeding of 2004 IEEE Conference on Cybernetics and

[12] L. Hui Intelligent Systems (IEEE, 2004), Vol. 1, pp. 340-344... "Multi-sensor imager registration and fusion," Ph.D. dissertation (University of California, 1993).

[13] E. Canga, "Image fusion," M.S. thesis (University of Bath, 2002).

[14] F. E. Ali, I. M. El-Dokany, A. A. Saad, and F. E. Abd ElSamie, "Curvelet fusion of MR and CT images," Prog. Electromagn.Res. C 3, 215-224 (2008).

[15] F. E. Ali, I. M. El-Dokany, A. A. Saad, and F. E. Abd ElSamie, "High resolution image acquisition from magnetic resonance and computed tomography scans using the curvelet fusion algorithm with inverse 
interpolation techniques" applied optics, Vol. 49, No. 1, pp. 114-125, 1 January 2010.

[16] E. A. Soltan, S. El-Rabaie, N. EL-Fishawy, F. E. Abd El-samie, S. E. Elkhamy, "Wavelet-Based Image Interpolation with A Least-squares Algorithm", computer engineering conference (ICENCO), 10.1109/ICENCO.2010.5720435, 2010, pp. 105-106.

[17] Y. Wang and B. Lohmann, "Multisensor image fusion: concept, method and applications," Tech. Rep., Institute of Automatic Technology, University of Bremen, Bremen, Germany, 2000.

[18] J. L. Starck, E. Candes, and D. L. Donoho, "The curvelet transform for image denoising," IEEE Trans. Image Process. 11, 670-684 (2002).

[19] G. Y. Chen and B. Kegl, "Image denoising with complex ridgelets,” Pattern Recogn. 578-585 (2007).

[20] J.-L. Starck, P. Abrial, Y. Moudden, and M. K. Nguyen, "Wavelets, ridgelets and curvelets on the sphere," Astron. Astrophys. 446, 1191-1204 (2006).

[21] F. E. Ali, I. M. El-Dokany, A. A. Saad, and F. E. Abd ElSamie, "Curvelet fusion of MR and CT images," Prog. Electromagn. Res. C 3, 215-224 (2008).
[22] C. Chao and J. Tao, "Study of image magnification based on curvelet transformation," in ISPRS Congress Beijing 2008, Proceedings of Commission II, The International Archives of the Photogrammetry, Remote Sensing, and Spatial Information Sciences (International Society for Photogrammetry and Remote Sensing, 2008), Vol. 37B2, pp. 289-292.

[23] J. Nunez, X. Otazu, O. Fors, A. Prades, V. Pala, and R. Arbiol, "Multi-resolution-based image fusion with additive wavelet decomposition," IEEE Trans. Geosci. Remote Sens. 37, 1204-1211 (1999).

[24] B. B. Saevarsson, J. R. Sveinsson, and J. A Benediktsson, "Combined wavelet and curvelet denoising of SAR images," in IEEE International Geoscience and Remote Sensing Symposium (IGARSS) (IEEE, 2004), Vol. 6, pp. 4235-4238.

[25] S. E. El-Khamy, M. M. Hadhoud, M. I. Dessouky, B. M. Salam, and F. E. Abd El-Samie, "Adaptive Leastsquares Acquisition of HR Images"International Journal of Information Acquisition (IJIA), March 2005.

[26] T. Kratochvil and P. Simicek, "Utilization of MATLAB for picture quality evaluation" ( Institute of Radio Electronics, Brno University of Technology, 2005). 